\title{
AMICA - the infrared eye at Dome C
}

\author{
Alberto Riva ${ }^{1}$, Mauro Dolci ${ }^{2}$, Oscar Straniero ${ }^{2}$, Filippo Maria Zerbi ${ }^{1}$, \\ Emilio Molinari ${ }^{1}$, Paolo Conconi ${ }^{1}$, Vincenzo De Caprio ${ }^{1}$, \\ Gaetano Valentini ${ }^{2}$, Gianluca Di Rico ${ }^{2}$, Maurizio Ragni ${ }^{2}$, \\ Danilo Pelusi ${ }^{2}$, Igor Di Varano ${ }^{2}$, Croce Giuliani ${ }^{2}$, Amico Di Cianno ${ }^{2}$, \\ Angelo Valentini ${ }^{2}$, Favio Bortoletto ${ }^{3}$, Maurizio D'Alessandro ${ }^{3}$, \\ Carlotta Bonoli ${ }^{3}$, Enrico Giro ${ }^{3}$, Daniela Fantinel ${ }^{3}$, Demetrio Magrin ${ }^{3}$, \\ Leonardo Corcione ${ }^{4}$, Maurizio Busso ${ }^{5}$, Gino Tosti ${ }^{5}$, \\ Giuliano Nucciarelli ${ }^{5}$, Fabio Roncella ${ }^{5}$, Carlos Abia ${ }^{6}$ \\ and the IRAIT Team

\footnotetext{
${ }^{1}$ INAF Osservatorio Astronomico di Brera - Merate, Merate, Italy

${ }^{2}$ INAF Osservatorio Astronomico Collurania Teramo, Teramo, Italy

${ }^{3}$ INAF Osservatorio Astronomico di Padova, Padova, Italy

${ }^{4}$ INAF Osservatorio Astronomico di Torino, Torino, Italy

${ }^{5}$ University of Perugia, Perugia, Italy

${ }^{6}$ University of Granada, Granada, Spain
}

\section{Introduction}

Amica (Antarctic Multiband Infrared Camera) is a dual-channel Infrared Imager (2$28 \mu \mathrm{m})$, that will be located at the Nasmyth focus of the IRAIT telescope at Dome C. Dome $\mathrm{C}$ base, on Antarctic plateau offers an unique chance for infrared astronomy. It has several advantages like temperature, pressure and site environment. Temperature, around $-60^{\circ} \mathrm{C}$ (mean) allows a good atmospheric stability (good seeing and good windows transparency) a low atmospheric background and the reduction of instrumental background. Pressure (equivalent of $4000 \mathrm{~m}$ a.s.l.), implies low content of water vapors; this means higher transmission, broader and new astronomical windows. The site offers the possibility of very long observations (about 6 months winter night).

\section{Science}

Most of the scientific targets of the IRAIT-AMICA project have been selected to take profit of the environmental conditions described above. Indeed the first goal is the site testing and characterization of the Dome $\mathrm{C}$ sky, in the bands between 2 to $28 \mu \mathrm{m}$, followed by a survey in these bands. Once the above general goals will be achieved a variety of cool IR targest could be observed with IRAIT-AMICA. Among these: AGB stars in the Milky Way, star formation regions in our and nearby galaxies, Solar System bodies, supernova remnants, extragalactic point-like sources, etc. Last, but not least, the IRAIT-AMICA collaboration will allow the development of the first permanent astronomical observatory in the Dome $\mathrm{C}$ base, with the possibility of find and solve a lot of environmental problems of a quasi-space situation.

\section{The instrument}

AMICA is a near-mid infrared camera, with a wavelength coverage between $2-28 \mu \mathrm{m}$. The camera has an all-reflective design (in order to reduce aberrations) and is composed by two off axis parabolas and two gold-coated plain mirrors. The wavelength coverage is done with two detectors: the SWA (Short Wavelength Array) that covers from 1 to $5.5 \mu \mathrm{m}$ (InSb, $258 \times 258$ pixels, Raytheon), and the LWA (Long Wavelength Array) that covers 
from 7 to $28 \mu \mathrm{m}$ (SiAs, $128 \times 128$ pixels, DRS Technologies). The instrument toggles between the LWA and the SWA by means of a folding mirror, before the detectors. The focal reduction of AMICA is 1:1.47 and the best sampling is at $3.42 \mu \mathrm{m}$ for SWA and $8.54 \mu \mathrm{m}$ for LWA. Plate scales are $0^{\prime \prime} .538 /$ pixel (SWA) and $1^{\prime \prime} .345 /$ pixel (LWA). The field of view is $2^{\prime} .29 \times 2^{\prime} .29$ for SWA and $2^{\prime} .87 \times 2^{\prime} .87$ for LWA. The entrance window is made of CdTe that guarantees and high transmission for all the $1-25 \mu \mathrm{m}$ microns band. The first-light set of filters will be a standard set in order to calibrate the camera with existing standards $\left(K, L, M, N_{1}, N_{2}, Q_{1}\right.$, and $\left.Q_{2}\right)$.

\section{The cryostat}

The internal temperature of the cryostat will be around $35 \mathrm{~K}$ except for the LWA detector that will be at $7 \mathrm{~K}$. All internal components (mirrors, optical bench, mountings, filterwheel, etc.) will be in aluminum, in order to have an homotetic contraction during the cooling-down process. This allows to align the optical components at room temperature and maintain the alignment at any temperature, drastically reducing the number of regulations and the necessity of human operations during the functioning time. The cryocooler will be an ARS 2-stage with a power consumption of $3.5 \mathrm{~kW}$. The choice of this element was a critical point: the power supply from the base is limited, and the low density of the atmosphere, causing limited convection, requires an increased capability of heat removal.

\section{Electronic and housekeeping}

Due to atmospheric conditions (temperature, pressure, etc.) we decided to keep only few elements at external temperature: the cryocooler head and the 'remote' unit. All the other subsystems will be placed in a temperature-controlled rack. Also for the electronic system we decided to divide the readout and control electronic in two subsections: the first is a 'remote unit' next to the telescope, with essential controls, the second is a 'local unit', inside the base. The software development has been designed as a modular structure, in order to drive both the telescope and the camera.

\section{Testing subsystems}

The AMICA team has designed and built ANTARES (ANTARctic Environment Simulator), a climatic chamber specifically designed to test each component before the delivery at Dome $\mathrm{C}$ of AMICA. ANTARES allows the test of small and medium size components with a pressure of $410 \mathrm{mbar}$, a temperature of $-60^{\circ} \mathrm{C}$ and relative humidity of $6 \%$.

\section{References}

Dolci, M., Straniero, O., Valentini, G., et al. 2006, SPIE, 6267, 48

Tosti, G., Busso, M., Nucciarelli, G., et al. 2006, SPIE, 6267, 47

Valentini, G., Magrin, D., Riva, A., et al. 2006, SPIE, 6267, 40

Bailey, J. 1996, PASA, 13, 7

Burton, M. G., Storey, J. W., \& Ashley, M. C. 2000, SPIE, 4005, 326B

Burton, M. G., Lawrence, J. S., Ashley, M. C. B., et al. 2005, PASA 22, 199

Chamberlain, M. A., Ashley, M. C. B., Burton, M. G., et al. 2000, ApJ, 535, 501

Harper, D. A. 1989, AIP-PC, 198, 123

Lawrence, J. S. 2004, PASP, 116, 482

Pel, J.-W., Glazenborg-Kluttig, A. W., de Haas, J. C., et al. 2000, SPIE, 4006, 164

Storey, J. W. V., Ashley, M. C. B., Lawrence, J. S., et al. 2003, Mem. S.A.It. Suppl., 2, 13

Walden, V. P., Town, M. S., Halter, B., \& Storey, J. W. V. 2005, PASP, 117, 300 